\title{
The Generation of Fullerenes
}

\author{
Gunnar Brinkmann \\ *Applied Mathematics \& Computer Science \\ Ghent University \\ Krijgslaan 281-S9, \\ 9000 Ghent, Belgium \\ Jan Goedgebeur \\ ${ }^{\dagger}$ Applied Mathematics \& Computer Science \\ Ghent University \\ Krijgslaan 281-S9, \\ 9000 Ghent, Belgium \\ Brendan D. McKay \\ ${ }^{\ddagger}$ Research School of Computer Science \\ Australian National University \\ ACT 0200, Australia
}

\begin{abstract}
We describe an efficient new algorithm for the generation of fullerenes. Our implementation of this algorithm is more than 3.5 times faster than the previously fastest generator for fullerenes - fullgen - and the first program since fullgen to be useful for more than 100 vertices. We also note a programming error in fullgen that caused problems for 136 or more vertices. We tabulate the numbers of fullerenes and IPR fullerenes up to 400 vertices. We also check up to 316 vertices a conjecture of Barnette that cubic planar graphs with maximum face size 6 are hamiltonian and verify that the smallest counterexample to the spiral conjecture has 380 vertices.

Note: this is the unedited version of our paper which was submitted and subsequently accepted for publication in Journal of Chemical Information and Modeling. The final edited and published version can be accessed at http://dx.doi.org/10.1021/ci3003107
\end{abstract}

\footnotetext{
*Gunnar.Brinkmann@UGent.be

†Jan.Goedgebeur@UGent.be

†bdm@cs.anu.edu.au
} 


\section{Introduction}

Fullerenes are spherical carbon molecules that can be modelled as cubic plane graphs where all faces are pentagons or hexagons. We will refer to these mathematical models also as fullerenes. Euler's formula implies that a fullerene with $n$ vertices contains exactly 12 pentagons and $n / 2-10$ hexagons. The dual of a fullerene is the plane graph obtained by exchanging the roles of vertices and faces: the vertex set of the dual graph is the set of faces of the original graph and two vertices in the dual graph are adjacent if and only if the two faces share an edge in the original graph. The rotational order around the vertices in the embedding of the dual fullerene follows the rotational order of the faces. As fullerenes and their duals are 3-connected, due to the theorem of Whitney the plane embeddings of fullerenes and duals of fullerenes are uniquely determined and the concept of graph isomorphism and isomorphism of embedded graphs (treating the mirror image as equivalent) coincide. The dual of a fullerene with $n$ vertices is a triangulation (i.e. every face is a triangle) which contains 12 vertices with degree 5 and $n / 2-10$ vertices with degree 6 .

Isolated Pentagon Rule (IPR) fullerenes are fullerenes where no two pentagons share an edge. IPR fullerenes are especially interesting due to a general tendency to be chemically more stable and thus more likely to occur in nature.

The first fullerene molecule was discovered in 1985 by Kroto et al. [12], namely the famous $C_{60}$ buckminsterfullerene or "buckyball". After that discovery several attempts have been made to generate complete lists of fullerene isomers.

The first approach was the spiral algorithm given by Manolopoulos et al. in 1991 [16. This algorithm was relatively inefficient and also incomplete in the sense that not every fullerene isomer could be generated with it. Manolopoulos and Fowler [15] gave an example of a fullerene that can not be constructed by this algorithm. The algorithm described here was the first to prove that the counterexample given by Manolopoulos and Fowler [15] is in fact smallest possible [6].

The spiral algorithm was later modified to make it complete, but the resulting algorithm was not efficient [14. In 1995 Yoshida and Osawa [19] proposed a different algorithm using folding nets, but its completeness has not been proven.

Other methods are described by Liu et al. [13] and Sah [18], but they also didn't lead to sufficiently efficient algorithms.

The most successful approach until now dates from 1997 and is given by Brinkmann and Dress [3]. The algorithm described there is proven to be complete and has been implemented in a program called fullgen. The basic strategy can be described as stitching together patches which are bounded by zigzag (Petrie) paths. Unfortunately a simple typo-like mistake in the 
source code produced an error that occurred for the first time at 136 vertices - far too many vertices to be detectable by any of the other programs until now. Due to this error the lists in the article of Brinkmann and Dress [3] contain some incorrect numbers which we will correct here.

The method of patch replacement can be described as replacing a finite connected region inside some fullerene with a larger patch with identical boundary. For energetical reasons, patch replacement as a chemical mechanism to grow fullerenes would need very small patches. Brinkmann et al. [4] investigated replacements of small patches and introduced two infinite families of operations. These operations can generate all fullerenes up to at least 200 vertices, but - as already shown in their paper - fail in general. In 2008 Hasheminezhad, Fleischner and McKay [10] described a recursive structure using patch replacements for the class of all fullerenes.

In section 2 of this paper we will describe an algorithm for the efficient generation of all non-isomorphic fullerenes using the construction operations from Hasheminezhad et al. [10]. In section 3 we will show how to extend this algorithm to generate only IPR fullerenes by using some simple look-aheads.

\section{Generation of fullerenes}

\subsection{The construction algorithm}

We call the patch replacement operations which replace a connected fragment of a fullerene by a larger fragment expansions and the inverse operations reductions. If $G^{\prime}$ is obtained from $G$ by an expansion, we call $G^{\prime}$ the child of $G$ and $G$ the parent of $G^{\prime}$.

From the results of Brinkmann et al. [7] it follows that no finite set of patch replacement operations is sufficient to construct all fullerenes from smaller ones. So each recursive structure based on patch replacement operations must necessarily allow an infinite number of different expansion types.

Hasheminezhad et al. [10] used two infinite families of expansions: $L_{i}$ and $B_{i, j}$ and a single expansion $F$. These expansions are sketched in Figure 1 . The lengths of the paths between the pentagons may vary and for operation $L_{i}$ the mirror image must also be considered. All faces drawn completely in the figure or labelled $f_{k}$ or $g_{k}$ have to be distinct. The faces labelled $f_{k}$ or $g_{k}$ can be either pentagons or hexagons, but when we refer to the pentagons of the operation, we always mean the two faces drawn as pentagons. For more details on the expansions see the article of Hasheminezhad et al. [10].

In Figure 2 the $L$ and $B$ expansions of Figure 1 are shown in dual representation. We will refer to vertices which have degree $k \in\{5,6\}$ in the dual representation of a fullerene as $k$-vertices. The solid white vertices in the figure are 5-vertices, the solid black vertices are 6-vertices and the shaded vertices can be either. 


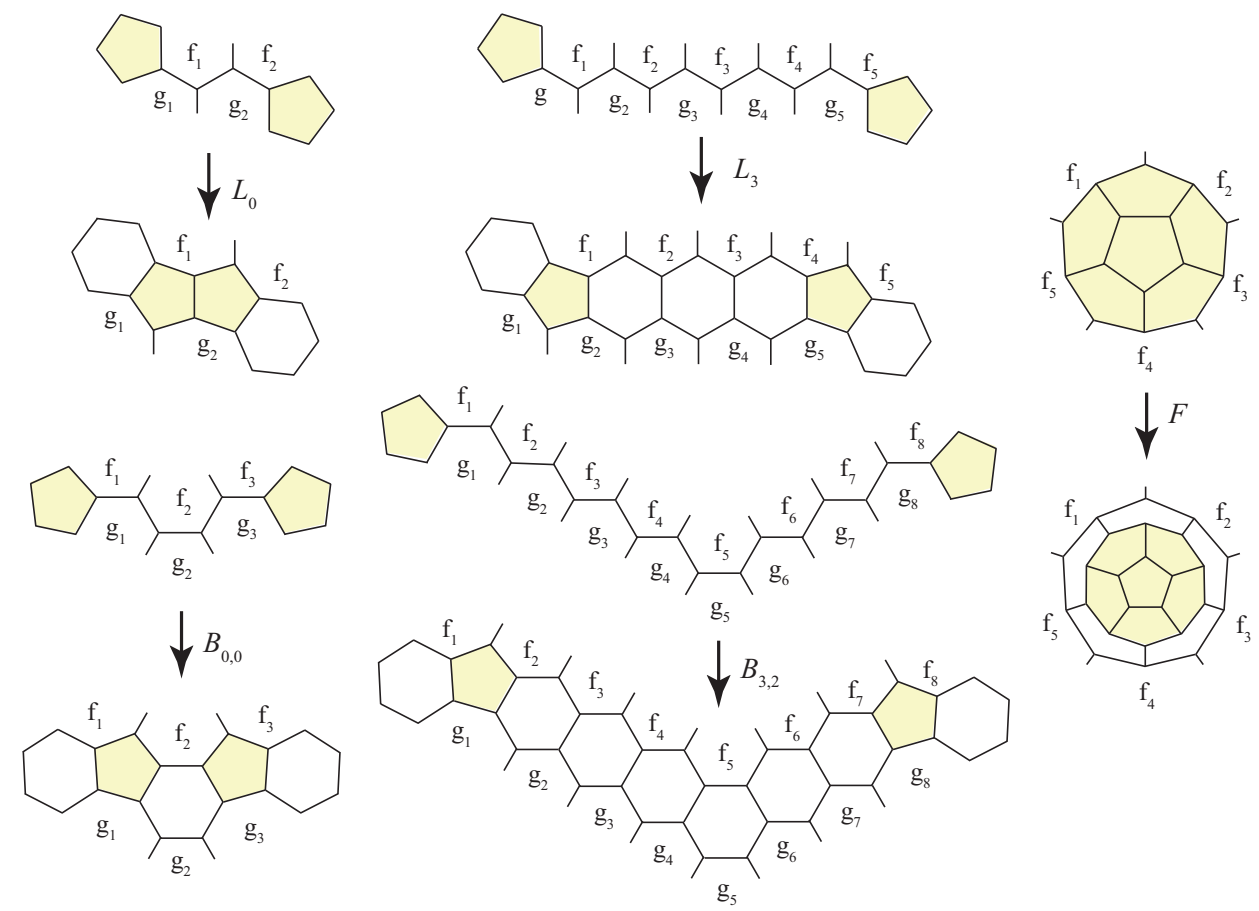

Figure 1: The $L, B$ and $F$ expansions for fullerenes.

Three special fullerenes $C_{20}$ (the dodecahedron), $C_{28}\left(T_{d}\right)$ and $C_{30}\left(D_{5 h}\right)$ are shown in Figure 3. The type-(5,0) nanotube fullerenes are those which can be made from $C_{30}\left(D_{5 h}\right)$ by applying expansion $F$ zero or more times. We will refer to all fullerenes not in one of these classes as reducible. The following theorem proved by Hasheminezhad et al. [10] shows that all reducible fullerenes can be reduced using a type $L$ or $B$ reduction.

Theorem 2.1. Every fullerene isomer, except $C_{28}\left(T_{d}\right)$ and type- $(5,0)$ nanotube fullerenes can be constructed by recursively applying expansions of type $L$ and $B$ to $C_{20}$.

Our algorithm uses this theorem by applying $L$ and $B$ expansions starting at $C_{20}$ and $C_{28}\left(T_{d}\right)$, together with separate (easy) computation of the type- $(5,0)$ nanotube fullerenes.

\subsection{Isomorphism rejection and optimizations}

If the expansions are applied in all possible ways, lots of isomorphic copies will be generated, but we wish to output only one example of each type. We use the canonical construction path method [17, but in the following we do not assume the reader to be familiar with the method.

In order to use this method, we first have to define a canonical reduction for every reducible dual fullerene $G$. This reduction must be unique up 

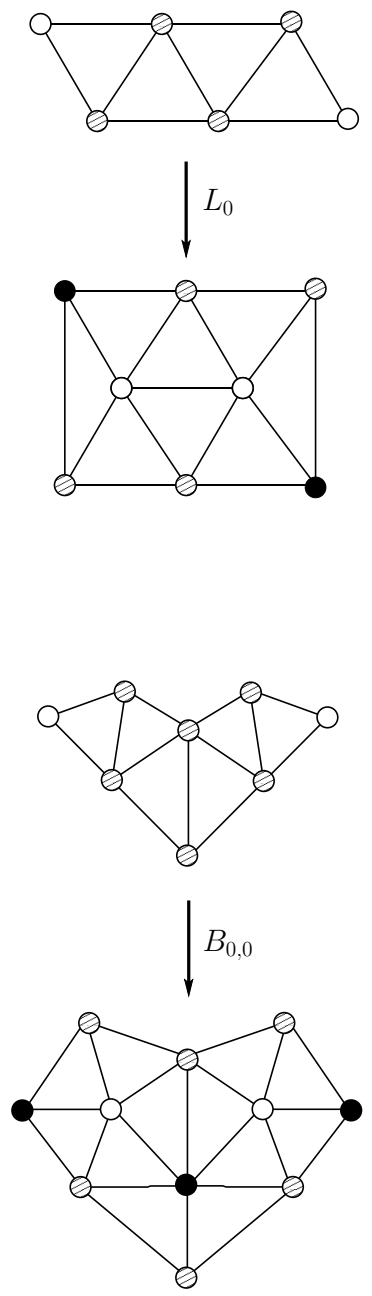

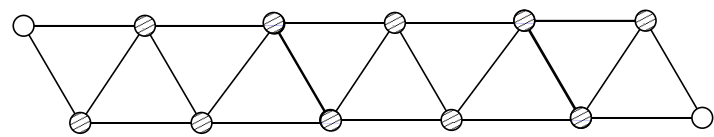

$L_{3}$
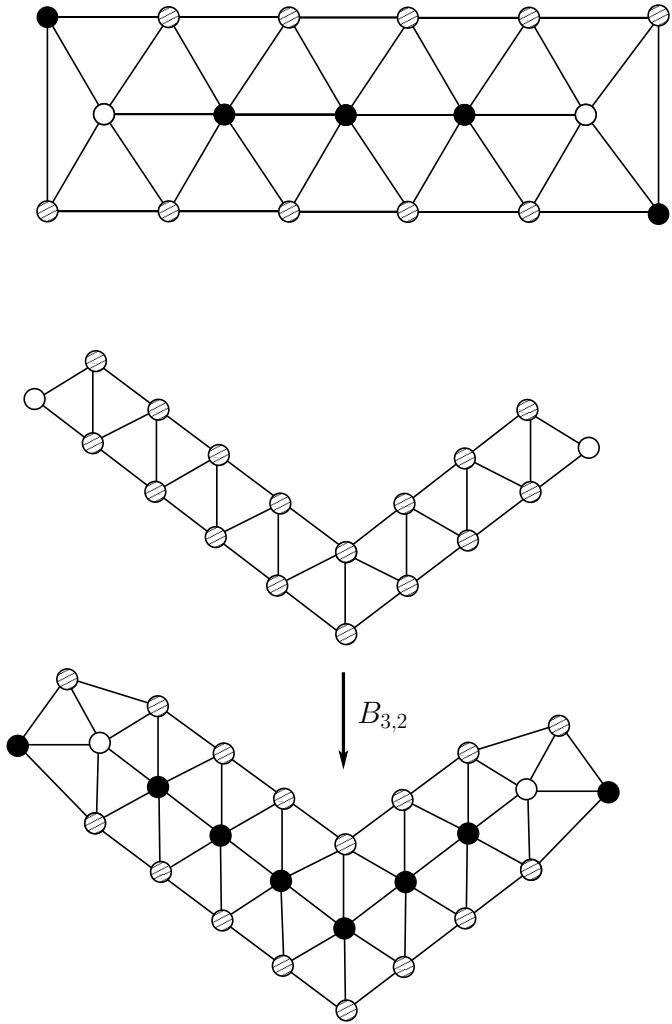

Figure 2: The $L$ and $B$ expansions in dual representation.

to automorphisms of $G$. We call the dual fullerene which is obtained by applying the canonical reduction to $G$ the canonical parent of $G$ and an expansion that is the inverse of a canonical reduction in the extended graph a canonical expansion.

We also define an equivalence relation on the set of all expansions or reductions of a given dual fullerene $G$. An expansion is completely characterized by the patch that is replaced with a larger patch. Two expansions are called equivalent if there is an automorphism of $G$ mapping the two corresponding patches onto each other. For reductions, the definition is similar, but in addition to the patch, a rotational direction is necessary to uniquely encode a reduction of type $L$. This direction can be a flag describing whether the new position of the pentagon is in clockwise or counterclockwise position of the path connecting the pentagons. Two type $L$ reductions are equivalent 


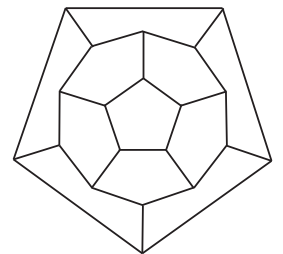

$\mathrm{C}_{20}$

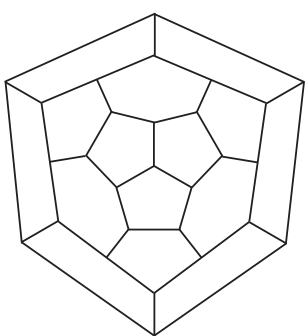

$\mathrm{C}_{28}\left(\mathrm{~T}_{\mathrm{d}}\right)$

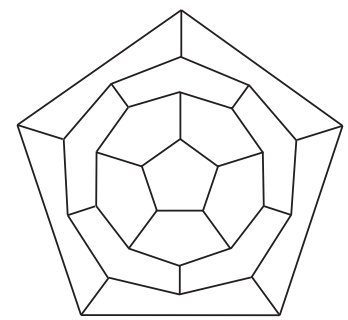

$\mathrm{C}_{30}\left(\mathrm{D}_{5 \mathrm{~h}}\right)$

Figure 3: The irreducible fullerenes.

if the patches are mapped onto each other by an orientation preserving automorphism and the flags are the same or they are mapped onto each other by an orientation reversing automorphism and the flags are different.

The two rules of the canonical construction path method applied to dual fullerenes are:

1. Only accept a dual fullerene if the last step in its construction was a canonical expansion.

2. For each dual fullerene $G$ to which expansions are applied, only apply one expansion from each equivalence class of expansions.

The expansions/reductions must of course be represented in an efficient way. Reductions are represented by triple $(e, x, d)$, where $e$ is a directed edge that is the first edge on the central path between the two pentagons, $x$ is the parameter set for the reduction (such as " $(2,3)$ " for $B_{2,3}$ ) and $d$ is a direction. For $B$ reductions, $d$ indicates whether the turn in the path is to the left or the right. For $L$ reductions, $d$ distinguishes between this reduction and its mirror image. Since $e$ can be at either end of the path, there are two equivalent triples for the same reduction, as illustrated in Figure 4. We call these triples the representing triples of the reduction. Expansions are also represented by triples in similar fashion.

When we translate the notion of equivalent reductions or expansions to representing triples, then the equivalence relation is generated by two relations. The first is that two triples are equivalent if they represent the same reduction. The second is that $(e, x, d)$ and $\left(e^{\prime}, x^{\prime}, d^{\prime}\right)$ are equivalent if $x=x^{\prime}$ and in case $d=d^{\prime}$ the edge $e$ can be mapped to $e^{\prime}$ by an orientation preserving automorphism and in case $d \neq d^{\prime}$ the edge $e$ can be mapped to $e^{\prime}$ by an orientation reversing automorphism.

For an efficient implementation of the canonicity criteria it is important that in many cases simple and easily computable criteria can decide on the 


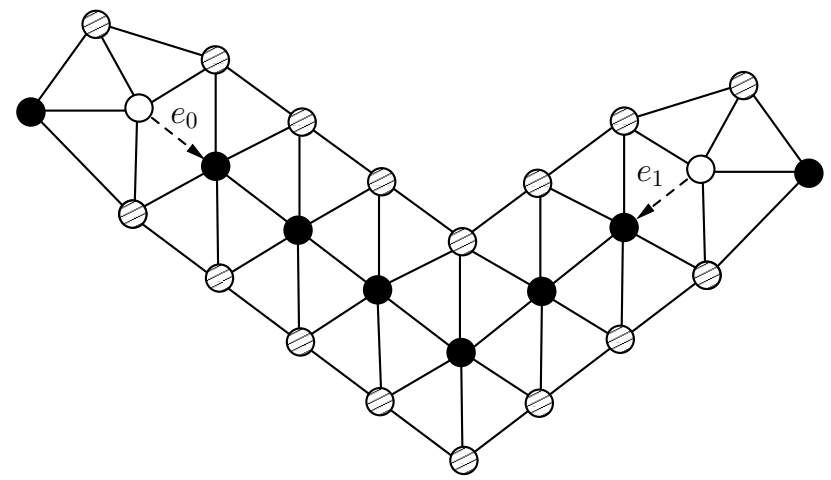

Figure 4: An example of two triples $\left(e_{0},(3,2), 1\right)$ and $\left(e_{1},(2,3), 0\right)$ representing the same $B$ reduction.

canonical reduction or at least reduce the list of possible reductions. To this end we assign a 6 -tuple $\left(x_{0}, \ldots, x_{5}\right)$ to every triple $(e, p, f)$ representing a possible reduction. We then choose the canonical reduction to be a reduction which has a representing triple with the smallest 6-tuple.

The values of $x_{0}, \ldots, x_{4}$ are combinatorial invariants of increasing discriminating power and cost. The value of $x_{0}$ is the length of the reduction of which $(e, p, f)$ is a representative. The length of the reduction is the distance between the two 5-vertices of the reduction before actually applying the reduction. So in case of a $B_{x, y}$ reduction (2 parameters) it is $x+y+2$ and in case of an $L_{x}$ reduction ( 1 parameter) it is $x+1$. Thus we give priority to short reductions. These are easier to detect and allow some look-ahead. The entry $x_{1}$ is the negative of the length of the longest straight path in the reduction. For an $L$ reduction, the value of $x_{1}$ is $-x_{0}$, which does not distinguish between two reductions with the same value of $x_{0}$. For a $B_{x, y}$ reduction it is $-\max \{x, y\}-1$, which sometimes distinguishes between $B$ reductions with the same value of $x_{0}$ and always distinguishes between an $L$ and a $B$ reduction with the same $x_{0}$.

The entries $x_{2}, x_{3}$ and $x_{4}$ are strings which contain the degrees of the vertices in well-defined neighbourhoods of the edge in the triple. These neighbourhoods are of increasing (constant) size.

In each case the value $x_{i}$ is only computed for those representing triples that have the smallest value of $\left(x_{0}, \ldots, x_{i-1}\right)$. As our main interest is whether an expansion we applied is canonical, we can also stop as soon as we have found a smaller 6-tuple, which may just mean a reduction with smaller value of $x_{0}$. In case of a unique triple with minimal value for $\left(x_{0}, \ldots, x_{i-1}\right)$ or two such triples representing the same reduction, we have found the canonical reduction and can stop the computation of the remaining values. If after the computation of $\left(x_{0}, \ldots, x_{4}\right)$ there is still more than one possibly canonical triple, we define $x_{5}$ as a string encoding the whole structure of the graph relative to the edge and the direction in the representing triple. See the 
article of Brinkmann and McKay [8] for details of this string, which can be in short be described as the code of a BFS-numbering starting at that edge and evaluating the neighbours of a vertex in the rotational order (clockwise/counterclockwise) given by the direction. Two triples coding patches in two graphs (that may be identical or not) containing the same directions are assigned the same value $x_{5}$ if and only if there is an orientation preserving isomorphism of the graphs mapping the edges in the triples onto each other. In case of different directions, the same value of $x_{5}$ is assigned if and only if there is such an orientation reversing automorphism. This final value $x_{5}$ makes sure that two patches (in the same or different graphs) with the same value of $\left(x_{0}, \ldots, x_{5}\right)$ can be mapped onto each other by an isomorphism $\phi()$ of the graph. When performing the corresponding reductions, the patches are replaced by smaller patches and replacing the images $\phi(v)$ of vertices inside the patch appropriately, one gets an isomorphism of the reduced graphs that maps the reduced patches onto each other.

When $x_{5}$ is computed and the graph $G$ that is tested for canonicity is accepted, as a byproduct we also have the automorphism group of $G$. As possible reductions are represented by edges starting at pentagons, we have a constant upper bound for the number of possible reductions to be evaluated. For a given triple, each of $x_{0}, \ldots, x_{4}$ can be computed in constant time and $x_{5}$ can be computed in linear time, so the canonicity test can be done in linear time.

Even though it is a nice feature that deciding canonicity of a given set of possible reductions can be done in linear time, for the practical performance it is more important that computing the combinatorial invariants $\left(x_{0}, \ldots, x_{4}\right)$ is of a small constant cost. For dual fullerenes with 152 vertices (fullerenes with 300 vertices), the discriminating power of $\left(x_{0}, \ldots, x_{4}\right)$ is enough to decide whether or not the last expansion was canonical in more than $99.9 \%$ of the cases.

In some cases these cheap invariants also allow look-aheads for deciding whether or not an expansion can be canonical before actually performing it. When making the lists of possible expansions, we can often already tell that a certain expansion cannot be canonical since it will not destroy all shorter reductions or since there will still be a reduction of the same length but with a smaller value for $x_{2}$. This avoids the application of a lot of noncanonical expansions. Counting only expansions passing this look-ahead, for dual fullerenes with 152 vertices still in $95.6 \%$ of the expansions a final decision can be found by only computing $\left(x_{0}, \ldots, x_{4}\right)$.

If there is only one representing triple with minimal value for $\left(x_{0}, \ldots, x_{i}\right)$ $(i \leq 4)$, the automorphism group of $G$ is trivial, so no extra computations are necessary. This happens in $80.9 \%$ of the cases for dual fullerenes with 152 vertices. The ratio is decreasing with the number of vertices. For 102 vertices of the dual fullerene it is $93.3 \%$ and for 127 vertices it is $86.9 \%$. 
Theorem 2.2. Assume that exactly one representative of each isomorphism class of dual fullerenes on up to $n-2$ vertices is given. Suppose we perform the following steps:

1. Perform one expansion of each equivalence class of $L$ and $B$ expansions which lead to a dual fullerene with $n$ vertices.

2. Accept each new dual fullerene if and only if a triple representing the inverse of the last expansion has the minimal value of $\left(x_{0}, \ldots, x_{5}\right)$ among all possible reductions.

Then exactly one representative of each isomorphism class of reducible dual fullerenes with $n$ vertices is accepted.

Proof. Let $G$ be a reducible dual fullerene with $n$ vertices. By Theorem 2.1 there is at least one reduction, and so a canonical reduction $\rho$, that applies to $G$. The graph resulting from $\rho$ is isomorphic to a graph in the input set, which has an expansion which is equivalent to the inverse of $\rho$. But this expansion produces a graph isomorphic to $G$ and the parameters of its inverse reduction are the same as those of $\rho$, so the result of the expansion is accepted.

This implies that at least one representative of each isomorphism class in question is generated. It remains to be shown that at most one is generated.

Suppose that the algorithm accepts two isomorphic fullerenes $G$ and $G^{\prime}$ with $n$ vertices. As they are isomorphic, the canonical reductions have the same parameter set $\left(x_{0}, \ldots, x_{5}\right)$. As they were both accepted, they were constructed by a canonical expansion, so - as mentioned before - the two parents $G_{0}$ and $G_{0}^{\prime}$ are isomorphic and there is an isomorphism that maps the corresponding expansions onto each other. By our assumption this means that $G_{0}$ and $G_{0}^{\prime}$ are identical and that the two expansions are equivalent, which contradicts step 1.

By recursively applying expansion $F$ to $C_{20}$, all type- $(5,0)$ fullerenes are constructed. As this constructs all type- $(5,0)$ fullerenes exactly once and these fullerenes can not be constructed by $L$ or $B$ expansions, this completes the algorithm.

\subsection{Optimizations}

As most fullerenes contain short reductions and as we give priority to short reductions, by far most long expansions are not canonical. For efficiency reasons it is interesting to determine an upper bound on the length of a canonical expansion.

Lemma 2.3. Reducible dual fullerenes which contain adjacent 5-vertices have an $L_{0}, L_{1}$ or $B_{0,0}$ reduction. 
Proof. For a proof, see the article of Hasheminezhad et al. [10].

So each reducible dual non-IPR fullerene has a reduction with length at most 2. In dual IPR fullerenes the shortest reduction is a reduction with the same length as the minimum distance of two 5 -vertices in the dual fullerene.

In dual fullerenes where the shortest distance between two 5 -vertices is at least $d$, the sets of vertices at distance at most $\left\lfloor\frac{d-1}{2}\right\rfloor$ of different vertices are disjoint. This gives us a lower bound of $12 f\left(\left\lfloor\frac{d-1}{2}\right\rfloor\right)$ for the number of vertices in the fullerene, where $f(x)=1+\frac{5}{2}(x+1) x$.

So expansions of length $d$ are not canonical if the expanded graph contains fewer than $12 f\left(\left\lfloor\frac{d-1}{2}\right\rfloor\right)$ vertices. This result does not only help to avoid the application of non-canonical expansions, but also avoids the need to search for long expansions.

We can often determine even sharper upper bounds for the maximum length of a canonical expansion:

Lemma 2.4. If a dual fullerene $G$ has a reduction of length $d \leq 2$, all children $G^{\prime}$ of $G$ have a reduction of length at most $d+2$.

Proof. If $G^{\prime}$ is not IPR, this follows from Lemma 2.3, so assume that $G^{\prime}$ is IPR. The length of the shortest reduction is then the shortest distance between two 5 -vertices. Let us look at the shortest path $W$ between two 5 -vertices allowing a reduction of length $d$ in $G$.

As $d \leq 2$ and as all vertices in the patch $P$ used for expansion must be distinct, $W$ can contain at most 2 maximal subpaths entering $P$ and ending there, starting in $P$ and leaving it or crossing $P$.

The distance between a 5 -vertex in $P$ from vertices on the boundary grows at most by 1 . The same is true for each pair of vertices on the boundary. So the path $W$ can grow in two places by at most 1, proving the result.

This lemma could be proven for larger $d$ if one required the child to be canonical, but as $12 f\left(\frac{5-1}{2}\right)=192$, all dual fullerenes with less than 192 vertices (or fullerenes with 380 vertices) have a reduction of length at most 4 . Therefore, even for $d=2$, Lemma 2.4 is only useful for fullerenes with at least 380 vertices.

Lemma 2.5. If a dual fullerene $G$ has an $L_{0}$ reduction, all canonical children $G^{\prime}$ of $G$ have a reduction of length at most 2.

Proof. If $G^{\prime}$ is not IPR, this follows from Lemma 2.3, so assume that $G^{\prime}$ is IPR. By Lemma 2.4. $G^{\prime}$ has a reduction of length at most 3, so a canonical child was constructed by an expansion of length at most 3. If $G^{\prime}$ was constructed by an $L_{0}, L_{1}$ or $B_{0,0}$ expansion, the statement follows immediately.

Figure 5 and Figure 6 show the only ways that an $L_{2}$ (resp. $B_{1,0}$ ) expansion can destroy an $L_{0}$ reduction which involves two pentagons $p_{1}$ and $p_{2}$ 
such that the expanded fullerene $G^{\prime}$ contains no reduction of length shorter than 3 . The faces $f_{i}$ and $g_{i}(1 \leq i \leq 4)$ which are on the boundary of the $L_{2}$ or $B_{1,0}$ expansion have to be hexagons otherwise the dual of $G^{\prime}$ would contain 5 -vertices which are at distance at most 2 . Since $p_{1}$ and $p_{2}$ are involved in the $L_{0}$ reduction, they must share an edge. So there is an edge $a \in\left\{e_{1}, e_{2}, e_{3}\right\}$ which is equal to an edge $b \in\left\{e_{4}, e_{5}, e_{6}\right\}$ and as the pentagons share an edge, they must also share two faces each containing an endpoint of this common edge. It is easy to see that for all possible choices of $a$ and $b$ this implies that a fullerene containing a patch from Figure 5 or Figure 6 must have a 4-edge-cut or a 5-edge-cut. However it follows from the results of Bornhöft et al. [1 that fullerenes are cyclically 5-edge connected, so 4-edge-cuts do not exist. Kardoš and S̆krekovski [11] showed that the type- $(5,0)$ nanotubes are the only fullerenes which have a non-trivial 5 -edge-cut.

So there is no expansion which can be applied to $G$ such that the shortest reduction of the expanded fullerene has length 3. Thus all canonical children of $G$ have a reduction of length at most 2 .

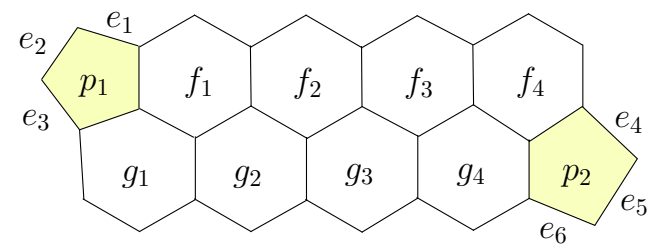

Figure 5: The initial patch of an $L_{2}$ expansion involving two neighbouring pentagons $p_{1}$ and $p_{2}$. One of the edges from $\left\{e_{1}, e_{2}, e_{3}\right\}$ is equal to an edge in $\left\{e_{4}, e_{5}, e_{6}\right\}$.

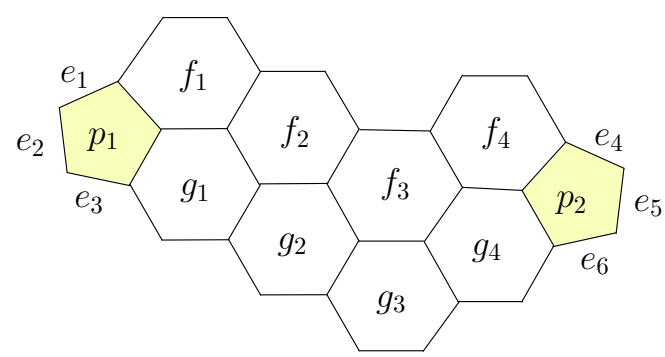

Figure 6: The initial patch of a $B_{1,0}$ expansion involving two neighbouring pentagons $p_{1}$ and $p_{2}$. One of the edges from $\left\{e_{1}, e_{2}, e_{3}\right\}$ is equal to an edge in $\left\{e_{4}, e_{5}, e_{6}\right\}$.

For the next lemmas the following observation is useful:

Observation 2.6. If the set of vertices contained in the initial patch of an expansion of length $l$ contains at least three 5-vertices (so in addition to the 
two 5-vertices of the expansion there is at least one more 5-vertex in the boundary), then in the extended patch there are two 5-vertices at distance at most $l / 2+1$.

Lemma 2.7. If a dual fullerene $G$ has at least two reductions of length 2 which do not have the same set of 5-vertices of the reduction, all canonical children $G^{\prime}$ have a reduction of length at most 3.

Proof. If $G^{\prime}$ is not a dual IPR fullerene, the result follows immediately, so assume the opposite. This implies that we have to find a bound for the minimum distance of two 5-vertices. By Lemma 2.4 each child has a reduction of length 4 . So each canonical child was constructed by an expansion of length at most 4 . If there were three 5 -vertices in the initial patch of the expansion, the result follows from Observation 2.6. So assume this is not the case and one 5-vertex of a reduction of length 2 is not contained in the initial patch. But then the distance to the other 5-vertex in the reduction can grow by at most 1 , proving the lemma.

Lemma 2.8. If a dual fullerene $G$ has at least three reductions of length 2 with pairwise disjoint sets of 5-vertices of the reduction, all canonical children $G^{\prime}$ of $G$ have a reduction of length at most 2.

Proof. We may again assume that $G^{\prime}$ is IPR. It follows from Lemma 2.7 that $G^{\prime}$ has a reduction of length at most 3 , so each canonical expansion has length at most 3 . If there are three 5 -vertices in the initial patch of the expansion, the result follows directly from the observation. So there is (at least) one reduction of length 2 so that none of its 5 -vertices is contained in that initial patch. But then the path of length 2 between these 5 -vertices still exists in the expanded graph and allows a reduction of length 2 .

For two reductions $R_{1}$ and $R_{2}$ in a dual fullerene $G$ we define the distance $d\left(R_{1}, R_{2}\right)$ to be $\min \left\{d\left(a_{1}, a_{2}\right) \mid a_{i}\right.$ is a 5 -vertex of $\left.R_{i}\right\}$.

Lemma 2.9. If a dual fullerene $G$ has $L_{0}$ reductions $R_{1}$ and $R_{2}$ with $d\left(R_{1}, R_{2}\right)>4$, all canonical children $G^{\prime}$ of $G$ have an $L_{0}$ reduction.

Proof. It follows from Lemma 2.5 that there is a reduction of length at most 2 in $G^{\prime}$. The distance between vertices which are in the initial patch of an expansion of length 2 is at most 4 . Therefore at least one of the two neighbouring 5 -vertex pairs still exists and the neighbouring vertices are either unchanged or changed to 6 -vertices. In either case the reduction will still be possible.

For dual fullerenes with 152 vertices, Lemmas 2.4, 2.7, 2.8 and 2.9 can be used to determine a bound on the length of canonical expansions in $93.9 \%$ of the cases. 


\section{Generation of IPR fullerenes}

The algorithm was developed for generating all fullerenes, but it can also be used to generate only IPR fullerenes by using a filter and some simple look-aheads:

An $L_{0}$ expansion is the only expansion that increases the number of vertices in a dual fullerene by just 2 vertices, but the result of an $L_{0}$ expansion is never a dual IPR-Fullerene. When constructing dual IPR fullerenes with $n$ vertices, dual IPR fullerenes with $n-2$ vertices do not have to be constructed and the largest dual fullerenes to which an expansion is applied have $n-3$ vertices.

For a dual fullerene with $n-4$ vertices only expansions of length 3 (i.e. $L_{2}$ or $B_{1,0}$ expansions) can lead to dual IPR fullerenes with $n$ vertices. However if a dual fullerene with $n-4$ vertices contains an $L_{0}$ reduction, it follows from Lemma 2.5 that expansions of length 3 are not canonical. Thus we can reject all dual fullerenes with $n-4$ vertices that contain an $L_{0}$ reduction and also avoid applying $L_{0}$ expansions to dual fullerenes with $n-6$ vertices.

Already these simple look-aheads result in an efficient program, as can be seen in Table 1 .

\section{Testing and results}

The running times and a comparison with fullgen are given in Table 1 . Our generator is called buckygen. The program was compiled with gcc and executed in a single thread on an Intel Xeon L5520 CPU at 2.27 GHz. The running times include writing the fullerenes to a null device.

Buckgen was used to generate all fullerenes up to 400 vertices. This led to a programming error being uncovered in fullgen that caused it to miss some fullerenes starting at 136 vertices and IPR fullerenes starting at 254 vertices. After correction of the error in fullgen, the two programs agree to at least 380 vertices, which is a good check of both. We give the counts in Tables 2 6, which correct those in the article of Brinkmann and Dress [3] where they overlap. The fullerenes themselves can be downloaded from http://hog.grinvin.org/Fullerenes for small sizes.

We also repeated and extended a computation reported by Brinkmann et al. [9], which relied on the faulty version of fullgen, the results are listed in Tables 2 5. Now we have confirmed that all cubic planar graphs with maximum face size 6 are hamiltonian to at least 316 vertices, in agreement with the famous conjecture of Barnette.

The incomplete lists of fullerenes were also used in another article of Brinkmann et al. [4]. All reducibility results given there remain true, except for Table 2, where the number of fullerenes that can not be reduced by a growth operation of cost 7 - that is replacing only 7 edges - is 1 too small 


\begin{tabular}{|c||c|c|c|c|}
\hline $\begin{array}{c}\text { number of } \\
\text { vertices }\end{array}$ & $\begin{array}{c}\text { fullerenes/s } \\
\text { (buckygen) }\end{array}$ & $\begin{array}{c}\text { fullgen (s) / } \\
\text { buckygen (s) }\end{array}$ & $\begin{array}{c}\text { IPR fullerenes/s } \\
\text { (buckygen) }\end{array}$ & $\begin{array}{c}\text { fullgen IPR (s) / } \\
\text { buckygen IPR (s) }\end{array}$ \\
\hline 100 & 42358 & 7.30 & 105 & 0.28 \\
140 & 33369 & 7.39 & 789 & 0.46 \\
170 & 21268 & 5.63 & 1174 & 0.58 \\
200 & 16953 & 5.49 & 1630 & 0.80 \\
230 & 12597 & 5.13 & 1721 & 0.96 \\
260 & 9408 & 4.59 & 1632 & 1.03 \\
280 & 7735 & 4.43 & 1530 & 1.10 \\
300 & 6494 & 4.07 & 1425 & 1.16 \\
320 & 5502 & 3.67 & 1332 & 1.14 \\
\hline $20-100$ & 159365 & 24.96 & 278 & 0.77 \\
$102-150$ & 157736 & 33.04 & 4643 & 2.44 \\
$152-200$ & 115625 & 32.08 & 10558 & 4.71 \\
$202-250$ & 82813 & 32.09 & 13212 & 6.84 \\
\hline
\end{tabular}

Table 1: Generation rates for fullerenes.

for 186 and 190 vertices and 2 too small for 194 vertices.

Our generator constructs larger fullerenes from smaller ones, so in order to generate all fullerenes with $n$ vertices, all fullerenes with at most $n-4$ vertices have to be generated as well (recall that an $L_{0}$ expansion increases the number of vertices by 4 ). So generating all fullerenes with at most $n$ vertices gives only a small overhead compared to generating all fullerenes with exactly $n$ vertices. In fullgen the overhead is considerably bigger as it does not construct fullerenes from smaller fullerenes. For example, buckygen can generate all fullerenes with $n \in[290,300]$ vertices more than 15 times faster than fullgen. More comparisons with fullgen can be found in Table1.

\section{$5 \quad$ Closing remarks}

We have described a new fullerene generator buckgen which is considerably faster than fullgen, which is the only previous generator capable of reaching 100 vertices. The generation cost is now likely to be lower than that of any significant computation performed on the generated structures.

After correction of an error in fullgen, we now have two independent counts of fullerenes up to 380 in full agreement, and values up to 400 vertices from buckgen.

The latest version of buckygen can be downloaded from [5]. Buckygen is also part of the $\mathrm{CaGe}$ software package [2]. 


\begin{tabular}{|c|c|c|c|c|c|}
\hline nv & nf & min. face 3 & min. face 4 & fullerenes & IPR fullerenes \\
\hline 4 & 4 & 1 & 0 & 0 & 0 \\
6 & 5 & 1 & 0 & 0 & 0 \\
8 & 6 & 1 & 1 & 0 & 0 \\
10 & 7 & 4 & 1 & 0 & 0 \\
12 & 8 & 8 & 2 & 0 & 0 \\
14 & 9 & 11 & 4 & 0 & 0 \\
16 & 10 & 23 & 7 & 0 & 0 \\
18 & 11 & 34 & 10 & 0 & 0 \\
20 & 12 & 54 & 22 & 1 & 0 \\
22 & 13 & 83 & 32 & 0 & 0 \\
24 & 14 & 125 & 58 & 1 & 0 \\
26 & 15 & 174 & 92 & 1 & 0 \\
28 & 16 & 267 & 151 & 2 & 0 \\
30 & 17 & 365 & 227 & 3 & 0 \\
32 & 18 & 509 & 368 & 6 & 0 \\
34 & 19 & 706 & 530 & 6 & 0 \\
36 & 20 & 963 & 805 & 15 & 0 \\
38 & 21 & 1270 & 1158 & 17 & 0 \\
40 & 22 & 1708 & 1695 & 40 & 0 \\
42 & 23 & 2204 & 2373 & 45 & 0 \\
44 & 24 & 2876 & 3354 & 89 & 0 \\
46 & 25 & 3695 & 4595 & 116 & 0 \\
48 & 26 & 4708 & 6340 & 199 & 0 \\
50 & 27 & 5925 & 8480 & 271 & 0 \\
52 & 28 & 7491 & 11417 & 437 & 0 \\
54 & 29 & 9255 & 15049 & 580 & 0 \\
56 & 30 & 11463 & 19832 & 924 & 0 \\
58 & 31 & 14083 & 25719 & 1205 & 0 \\
60 & 32 & 17223 & 33258 & 1812 & 1 \\
62 & 33 & 20857 & 42482 & 2385 & 0 \\
64 & 34 & 25304 & 54184 & 3465 & 0 \\
66 & 35 & 30273 & 68271 & 4478 & 0 \\
68 & 36 & 36347 & 85664 & 6332 & 0 \\
70 & 37 & 43225 & 106817 & 8149 & 1 \\
72 & 38 & 51229 & 132535 & 11190 & 1 \\
74 & 39 & 60426 & 163194 & 14246 & 1 \\
76 & 40 & 71326 & 200251 & 19151 & 2 \\
78 & 41 & 83182 & 244387 & 24109 & 5 \\
80 & 42 & 97426 & 296648 & 31924 & 7 \\
82 & 43 & 113239 & 358860 & 39718 & 9 \\
84 & 44 & 131425 & 431578 & 51592 & 24 \\
86 & 45 & 151826 & 517533 & 63761 & 19 \\
88 & 46 & 175302 & 617832 & 81738 & 35 \\
\hline & & & & & \\
\hline
\end{tabular}

Table 2: Cubic plane graphs with maximum face size 6 listed with respect to their minimum face size. Cubic plane graphs with maximum face size 6 and with minimum face size 5 are fullerenes. nv is the number of vertices and $\mathrm{nf}$ is the number of faces. 


\begin{tabular}{|c|c|c|c|c|c|}
\hline nv & $\mathrm{nf}$ & $\min$. face 3 & $\min$. face 4 & fullerenes & IPR fullerenes \\
\hline 90 & 47 & 200829 & 735257 & 99918 & 46 \\
\hline 92 & 48 & 231042 & 870060 & 126409 & 86 \\
\hline 94 & 49 & 263553 & 1029114 & 153493 & 134 \\
\hline 96 & 50 & 300602 & 1209783 & 191839 & 187 \\
\hline 98 & 51 & 341960 & 1420472 & 231017 & 259 \\
\hline 100 & 52 & 388673 & 1659473 & 285914 & 450 \\
\hline 102 & 53 & 438795 & 1937509 & 341658 & 616 \\
\hline 104 & 54 & 496961 & 2249285 & 419013 & 823 \\
\hline 106 & 55 & 559348 & 2612410 & 497529 & 1233 \\
\hline 108 & 56 & 629807 & 3015386 & 604217 & 1799 \\
\hline 110 & 57 & 706930 & 3483289 & 713319 & 2355 \\
\hline 112 & 58 & 792703 & 4002504 & 860161 & 3342 \\
\hline 114 & 59 & 885137 & 4600343 & 1008444 & 4468 \\
\hline 116 & 60 & 990929 & 5257856 & 1207119 & 6063 \\
\hline 118 & 61 & 1102609 & 6019580 & 1408553 & 8148 \\
\hline 120 & 62 & 1227043 & 6849385 & 1674171 & 10774 \\
\hline 122 & 63 & 1363825 & 7805813 & 1942929 & 13977 \\
\hline 124 & 64 & 1513612 & 8846570 & 2295721 & 18769 \\
\hline 126 & 65 & 1673568 & 10041875 & 2650866 & 23589 \\
\hline 128 & 66 & 1853928 & 11335288 & 3114236 & 30683 \\
\hline 130 & 67 & 2045154 & 12821597 & 3580637 & 39393 \\
\hline 132 & 68 & 2255972 & 14415241 & 4182071 & 49878 \\
\hline 134 & 69 & 2485363 & 16248586 & 4787715 & 62372 \\
\hline 136 & 70 & 2732106 & 18211371 & 5566949 & 79362 \\
\hline 138 & 71 & 2998850 & 20454114 & 6344698 & 98541 \\
\hline 140 & 72 & 3295090 & 22845387 & 7341204 & 121354 \\
\hline 142 & 73 & 3606102 & 25587469 & 8339033 & 151201 \\
\hline 144 & 74 & 3944923 & 28486985 & 9604411 & 186611 \\
\hline 146 & 75 & 4316999 & 31808776 & 10867631 & 225245 \\
\hline 148 & 76 & 4711038 & 35313026 & 12469092 & 277930 \\
\hline 150 & 77 & 5135794 & 39315258 & 14059174 & 335569 \\
\hline 152 & 78 & 5599065 & 43529295 & 16066025 & 404667 \\
\hline 154 & 79 & 6091434 & 48339505 & 18060979 & 489646 \\
\hline 156 & 80 & 6621013 & 53361979 & 20558767 & 586264 \\
\hline 158 & 81 & 7198926 & 59117693 & 23037594 & 697720 \\
\hline 160 & 82 & 7800960 & 65110208 & 26142839 & 836497 \\
\hline 162 & 83 & 8460776 & 71938170 & 29202543 & 989495 \\
\hline 164 & 84 & 9168333 & 79041733 & 33022573 & 1170157 \\
\hline 166 & 85 & 9917772 & 87147815 & 36798433 & 1382953 \\
\hline 168 & 86 & 10711603 & 95517631 & 41478344 & 1628029 \\
\hline 170 & 87 & 11590680 & 105090752 & 46088157 & 1902265 \\
\hline 172 & 88 & 12491734 & 114936807 & 51809031 & 2234133 \\
\hline 174 & 89 & 13479003 & 126169808 & 57417264 & 2601868 \\
\hline 176 & 90 & 14518882 & 137732548 & 64353269 & 3024383 \\
\hline
\end{tabular}

Table 3: Cubic plane graphs with maximum face size 6 listed with respect to their minimum face size (continued). $\mathrm{nv}$ is the number of vertices and $\mathrm{nf}$ is the number of faces. 


\begin{tabular}{|c|c|c|c|c|}
\hline $\mathrm{nv}$ & $\mathrm{nf}$ & $\min$. face 4 & fullerenes & IPR fullerenes \\
\hline 178 & 91 & 150895768 & 71163452 & 3516365 \\
\hline 180 & 92 & 164343840 & 79538751 & 4071832 \\
\hline 182 & 93 & 179752024 & 87738311 & 4690880 \\
\hline 184 & 94 & 195420760 & 97841183 & 5424777 \\
\hline 186 & 95 & 213287269 & 107679717 & 6229550 \\
\hline 188 & 96 & 231489614 & 119761075 & 7144091 \\
\hline 190 & 97 & 252233869 & 131561744 & 8187581 \\
\hline 192 & 98 & 273226069 & 145976674 & 9364975 \\
\hline 194 & 99 & 297264792 & 159999462 & 10659863 \\
\hline 196 & 100 & 321450554 & 177175687 & 12163298 \\
\hline 198 & 101 & 349098672 & 193814658 & 13809901 \\
\hline 200 & 102 & 376999869 & 214127742 & 15655672 \\
\hline 202 & 103 & 408774872 & 233846463 & 17749388 \\
\hline 204 & 104 & 440627726 & 257815889 & 20070486 \\
\hline 206 & 105 & 477200827 & 281006325 & 22606939 \\
\hline 208 & 106 & 513632380 & 309273526 & 25536557 \\
\hline 210 & 107 & 555304108 & 336500830 & 28700677 \\
\hline 212 & 108 & 596974072 & 369580714 & 32230861 \\
\hline 214 & 109 & 644526803 & 401535955 & 36173081 \\
\hline 216 & 110 & 691786828 & 440216206 & 40536922 \\
\hline 218 & 111 & 746085995 & 477420176 & 45278722 \\
\hline 220 & 112 & 799648739 & 522599564 & 50651799 \\
\hline 222 & 113 & 861133064 & 565900181 & 56463948 \\
\hline 224 & 114 & 922082216 & 618309598 & 62887775 \\
\hline 226 & 115 & 991650902 & 668662698 & 69995887 \\
\hline 228 & 116 & 1060208550 & 729414880 & 77831323 \\
\hline 230 & 117 & 1139239947 & 787556069 & 86238206 \\
\hline 232 & 118 & 1216496915 & 857934016 & 95758929 \\
\hline 234 & 119 & 1305306936 & 925042498 & 105965373 \\
\hline 236 & 120 & 1392596607 & 1006016526 & 117166528 \\
\hline 238 & 121 & 1492525091 & 1083451816 & 129476607 \\
\hline 240 & 122 & 1590214959 & 1176632247 & 142960479 \\
\hline 242 & 123 & 1702998124 & 1265323971 & 157402781 \\
\hline 244 & 124 & 1812247954 & 1372440782 & 173577766 \\
\hline 246 & 125 & 1938356975 & 1474111053 & 190809628 \\
\hline
\end{tabular}

Table 4: Triangle-free cubic plane graphs with maximum face size 6 listed with respect to their minimum face size. nv is the number of vertices and $\mathrm{nf}$ is the number of faces. 


\begin{tabular}{|c|c|c|c|c|}
\hline nv & $\mathrm{nf}$ & $\min$. face 4 & fullerenes & IPR fullerenes \\
\hline 248 & 126 & 2061311003 & 1596482232 & 209715141 \\
\hline 250 & 127 & 2202202308 & 1712934069 & 230272559 \\
\hline 252 & 128 & 2338869735 & 1852762875 & 252745513 \\
\hline 254 & 129 & 2497257527 & 1985250572 & 276599787 \\
\hline 256 & 130 & 2649382974 & 2144943655 & 303235792 \\
\hline 258 & 131 & 2825361014 & 2295793276 & 331516984 \\
\hline 260 & 132 & 2995557818 & 2477017558 & 362302637 \\
\hline 262 & 133 & 3191292821 & 2648697036 & 395600325 \\
\hline 264 & 134 & 3379722482 & 2854536850 & 431894257 \\
\hline 266 & 135 & 3598542661 & 3048609900 & 470256444 \\
\hline 268 & 136 & 3806922124 & 3282202941 & 512858451 \\
\hline 270 & 137 & 4049087424 & 3501931260 & 557745670 \\
\hline 272 & 138 & 4281540754 & 3765465341 & 606668511 \\
\hline 274 & 139 & 4549259510 & 4014007928 & 659140287 \\
\hline 276 & 140 & 4805073991 & 4311652376 & 716217922 \\
\hline 278 & 141 & 5103457703 & 4591045471 & 776165188 \\
\hline 280 & 142 & 5385296261 & 4926987377 & 842498881 \\
\hline 282 & 143 & 5713728893 & 5241548270 & 912274540 \\
\hline 284 & 144 & 6026548238 & 5618445787 & 987874095 \\
\hline 286 & 145 & 6388285729 & 5972426835 & 1068507788 \\
\hline 288 & 146 & 6731485975 & 6395981131 & 1156161307 \\
\hline 290 & 147 & 7132734985 & 6791769082 & 1247686189 \\
\hline 292 & 148 & 7508699038 & 7267283603 & 1348832364 \\
\hline 294 & 149 & 7948994131 & 7710782991 & 1454359806 \\
\hline 296 & 150 & 8365304423 & 8241719706 & 1568768524 \\
\hline 298 & 151 & 8847679520 & 8738236515 & 1690214836 \\
\hline 300 & 152 & 9302042370 & 9332065811 & 1821766896 \\
\hline 302 & 153 & 9835862103 & 9884604767 & 1958581588 \\
\hline 304 & 154 & 10332102625 & 10548218751 & 2109271290 \\
\hline 306 & 155 & 10915020041 & 11164542762 & 2266138871 \\
\hline 308 & 156 & 11462133758 & 11902015724 & 2435848971 \\
\hline 310 & 157 & 12098825145 & 12588998862 & 2614544391 \\
\hline 312 & 158 & 12694519224 & 13410330482 & 2808510141 \\
\hline 314 & 159 & 13396207247 & 14171344797 & $\begin{array}{llll}3 & 009 & 120 & 113\end{array}$ \\
\hline 316 & 160 & 14043402497 & 15085164571 & 3229731630 \\
\hline
\end{tabular}

Table 5: Triangle-free cubic plane graphs with maximum face size 6 listed with respect to their minimum face size (continued). nv is the number of vertices and $\mathrm{nf}$ is the number of faces. 


\begin{tabular}{|c|c|c|c|}
\hline nv & $\mathrm{nf}$ & fullerenes & IPR fullerenes \\
\hline 318 & 161 & 15930619304 & 3458148016 \\
\hline 320 & 162 & 16942010457 & 3704939275 \\
\hline 322 & 163 & 17880232383 & 3964153268 \\
\hline 324 & 164 & 19002055537 & 4244706701 \\
\hline 326 & 165 & 20037346408 & 4533465777 \\
\hline 328 & 166 & 21280571390 & 4850870260 \\
\hline 330 & 167 & 22426253115 & 5178120469 \\
\hline 332 & 168 & 23796620378 & 5531727283 \\
\hline 334 & 169 & 25063227406 & 5900369830 \\
\hline 336 & 170 & 26577912084 & 6299880577 \\
\hline 338 & 171 & 27970034826 & 6709574675 \\
\hline 340 & 172 & 29642262229 & 7158963073 \\
\hline 342 & 173 & 31177474996 & 7620446934 \\
\hline 344 & 174 & 33014225318 & 8118481242 \\
\hline 346 & 175 & 34705254287 & 8636262789 \\
\hline 348 & 176 & 36728266430 & 9196920285 \\
\hline 350 & 177 & 38580626759 & 9768511147 \\
\hline 352 & 178 & 40806395661 & 10396040696 \\
\hline 354 & 179 & 42842199753 & 11037658075 \\
\hline 356 & 180 & 45278616586 & 11730538496 \\
\hline 358 & 181 & 47513679057 & 12446446419 \\
\hline 360 & 182 & 50189039868 & 13221751502 \\
\hline 362 & 183 & 52628839448 & 14010515381 \\
\hline 364 & 184 & 55562506886 & 14874753568 \\
\hline 366 & 185 & 58236270451 & 15754940959 \\
\hline 368 & 186 & 61437700788 & 16705334454 \\
\hline 370 & 187 & 64363670678 & 17683643273 \\
\hline 372 & 188 & 67868149215 & 18744292915 \\
\hline 374 & 189 & 71052718441 & 19816289281 \\
\hline 376 & 190 & 74884539987 & 20992425825 \\
\hline 378 & 191 & 78364039771 & 22186413139 \\
\hline 380 & 192 & 82532990559 & 23475079272 \\
\hline 382 & 193 & 86329680991 & 24795898388 \\
\hline 384 & 194 & $90881 \quad 152117$ & 26227197453 \\
\hline 386 & 195 & 95001297565 & 27670862550 \\
\hline 388 & 196 & 99963147805 & 29254036711 \\
\hline 390 & 197 & 104453597992 & 30852950986 \\
\hline 392 & 198 & 109837310021 & 32581366295 \\
\hline 394 & 199 & 114722988623 & 34345173894 \\
\hline 396 & 200 & 120585261143 & $\begin{array}{lll}36 & 259 & 212641\end{array}$ \\
\hline 398 & 201 & 125873325588 & 38179777473 \\
\hline 400 & 202 & 132247999328 & 40286153024 \\
\hline
\end{tabular}

Table 6: Counts of fullerenes and IPR fullerenes. nv is the number of vertices and $\mathrm{nf}$ is the number of faces. 


\subsection{Acknowledgements}

This work was carried out using the Stevin Supercomputer Infrastructure at Ghent University. Jan Goedgebeur is supported by a PhD grant from the Research Foundation of Flanders (FWO). Brendan McKay is supported by the Australian Research Council.

\section{References}

[1] J. Bornhöft, G. Brinkmann, and J. Greinus. Pentagonhexagon-patches with short boundaries. European Journal of Combinatorics, 24(5):517$529,2003$.

[2] G. Brinkmann, O. Delgado Friedrichs, S. Lisken, A. Peeters, and N. Van Cleemput. CaGe - a Virtual Environment for Studying Some Special Classes of Plane Graphs - an Update. MATCH Commun. Math. Comput. Chem., 63(3):533-552, 2010. Available at http://caagt. ugent.be/CaGe.

[3] G. Brinkmann and A.W.M. Dress. A constructive enumeration of fullerenes. Journal of Algorithms, 23:345-358, 1997.

[4] G. Brinkmann, D. Franceus, P.W. Fowler, and J.E. Graver. Growing fullerenes from seed: Growth transformations of fullerene polyhedra. Chemical Physics Letters, 428:386-393, 2006.

[5] G. Brinkmann, J. Goedgebeur, and B.D. McKay. Homepage of buckygen: http://caagt.ugent.be/buckygen/.

[6] G. Brinkmann, J. Goedgebeur, and B.D. McKay. The smallest fullerene without a spiral. Chemical Physics Letters, 522(2):54-55, 2012.

[7] G. Brinkmann, J.E. Graver, and C. Justus. Numbers of faces in disordered patches. Journal of Mathematical Chemistry, 45(2):263-278, 2009.

[8] G. Brinkmann and B.D. McKay. Fast generation of planar graphs. MATCH Commun. Math. Comput. Chem., 58(2):323-357, 2007.

[9] G. Brinkmann, B.D. McKay, and U. von Nathusius. Backtrack search and look-ahead for the construction of planar cubic graphs with restricted face sizes. MATCH Commun. Math. Comput. Chem., 48:163177, 2003.

[10] M. Hasheminezhad, H. Fleischner, and B.D. McKay. A universal set of growth operations for fullerenes. Chemical Physics Letters, 464:118$121,2008$. 
[11] F. Kardoš and R. S̆krekovski. Cyclic edge-cuts in fullerene graphs. Journal of Mathematical Chemistry, 44(1):121-132, 2008.

[12] H.W. Kroto, J.R. Heath, S.C. O'Brien, R.F. Curl, and R.E. Smalley. $C_{60}$ : Buckminsterfullerene. Nature, 318:162-163, 1985.

[13] X. Liu, D.J. Klein, T.G. Schmalz, and W.A. Seitz. Generation of carbon cage polyhedra. Journal of Computational Chemistry, 12(10):1252$1259,1991$.

[14] D.E. Manolopoulos and P.W. Fowler. Molecular graphs, point groups, and fullerenes. Journal of Chemical Physics, 96(10):7603-7614, 1992.

[15] D.E. Manolopoulos and P.W. Fowler. A fullerene without a spiral. Chemical Physics Letters, 204(1-2):1-7, 1993.

[16] D.E. Manolopoulos and J.C. May. Theoretical studies of the fullerenes: $C_{34}$ to $C_{70}$. Chemical Physics Letters, 181:105-111, 1991.

[17] B.D. McKay. Isomorph-free exhaustive generation. Journal of Algorithms, 26(2):306-324, 1998.

[18] C.H. Sah. Combinatorial construction of fullerene structures. Croatica Chemica Acta, 66:1-12, 1993.

[19] M. Yoshida and E. Osawa. Formalized drawing of fullerene nets. 1. algorithm and exhaustive generation of isomeric structures. Bulletin of the Chemical Society of Japan, 68:2073-2081, 1995. 